\title{
Robot-assisted surgery in gynecological oncology: a review
}

This article was published in the following Dove Press journal:

Robotic Surgery: Research and Reviews

23 September 2015

Number of times this article has been viewed

\section{Eric C Liberman \\ Gary L Goldberg \\ Dennis Yi-Shin Kuo}

Department of Obstetrics and Gynecology and Women's Health, Albert Einstein College of Medicine, Montefiore Medical Center, Bronx, NY, USA
Correspondence: Gary L Goldberg Department of Obstetrics and Gynecology and Women's Health, Albert Einstein College of Medicine, 1300 Morris Park Avenue, Belfer Educational Center, Room 50I, Bronx, NY 1046I, USA

$\mathrm{Tel}+\mathrm{I} 7184302588$

Fax +I 7184302365

Email ggoldber@montefiore.org
Abstract: Robot-assisted surgery has forever changed the surgical management of gynecologic malignancies since it first gained approval by the US Food and Drug Administration in 2005 for use in gynecological surgery. Robot-assisted surgery has enabled a greater number of gynecologic oncologists to offer their patients minimally invasive procedures that were once performed mostly by laparotomy and only rarely by traditional laparoscopy. Although traditional laparoscopy has been present in our field for several decades, its current utilization pales in comparison to that of robot-assisted surgery. Robot-assisted surgery is currently being utilized in the management of endometrial, cervical, and ovarian cancers, and its implementation in more complex procedures is expanding at a rapid pace. The purpose of this review article is to evaluate the current literature and provide a critical appraisal of the current indications, perioperative outcomes, and the future direction of robot-assisted surgery in gynecologic oncology. A PubMed database search was performed using keywords such as "robotic surgery", "endometrial cancer", "cervical cancer", and "ovarian cancer". Relevant articles were reviewed and incorporated as deemed appropriate.

Keywords: gynecological cancer, laparoscopic surgery, gynecological surgery, da Vinci robot, minimally invasive surgery

\section{Introduction}

Over the last decade, gynecologic oncologists have increasingly utilized minimally invasive surgical techniques to manage and stage gynecologic cancers. This is especially true with regard to the use of robot-assisted surgery by gynecologic oncologists. In a 2015 survey of members of the Society of Gynecologic Oncology (SGO), ${ }^{1} 406$ (32\%) of the 1,279 SGO members responded through an online or mailed-paper survey. Eightythree percent of the respondents $(n=337)$ performed traditional laparoscopic surgery (compared with 84\% in 2004 and 91\% in 2007). Ninety-seven percent of respondents performed robot-assisted surgery (compared with 27\% in 2007). Respondents were asked to indicate which procedures they performed utilizing robot-assisted surgery but not with traditional laparoscopy, and 75\% indicated radical hysterectomy and pelvic lymphadenectomy for cervical cancer. Overall, 70\% of the respondents indicated that hysterectomy and staging for uterine cancer was the surgical procedure they most commonly performed using a minimally invasive approach. The authors concluded there was a significant increase in the overall use and indications for robot-assisted surgery.

Robot-assisted surgery in gynecological oncology has expanded since first being approved for use in gynecological surgery by the US Food and Drug Administration 
(FDA) in 2005. As of June 30, 2015, there were 3,398 robotic platforms worldwide with 2,295 in the United States, 573 in Europe, 206 in Japan, and 324 in the rest of the world. ${ }^{2}$ In 2007 , there were $\sim 800$ robotic platforms in the USA and 200 in all other countries combined. ${ }^{3}$

Another survey published in 2010 revealed that $95 \%$ of gynecologic oncology fellows have a robotic platform at their institutions, and 95\% were trained to use it. ${ }^{4}$ The SGO's robotic task force position statement in 2012 indicated that robot-assisted surgery has indeed markedly changed the practice patterns in the US gynecologic oncologist community and robot-assisted surgery has had a significant impact on the minimally invasive surgical approach to patients with gynecologic malignancies in the USA. ${ }^{5}$ Additionally, this task force recommended that fellowship programs should include standardized training in both robot-assisted surgery and traditional laparoscopic surgery. Fellowship directors and fellows-in-training generally have a favorable view of this evolving technology. Based on these responses, robotassisted surgery will play an increasingly important role in the future. ${ }^{4}$

The advantages of minimally invasive surgery over laparotomy are clear and include improved perioperative outcomes, shorter hospital stays, better cosmetic results, improved quality of life, and a quicker return to daily functions and the workforce. ${ }^{6}$ Within the minimally invasive surgical realm, there are apparent advantages of robot-assisted surgery over laparoscopy. The advantages of robot-assisted surgery over laparoscopy include high-definition three-dimensional field of vision, instruments with wrist-like range of motion, tremor filtration, better ergonomics, and a faster learning curve compared to traditional laparoscopy. ${ }^{7-9}$ However, there are potential disadvantages to robot-assisted surgery, including the perceived increased cost, loss of haptics, and lack of prospective studies.

While many of the advantages and disadvantages of robot-assisted surgery are perceived, there is a paucity of randomized controlled trials demonstrating whether or not robot-assisted surgery is clinically superior to the other surgical modalities. Does the use of robot-assisted surgery improve the clinical outcomes in women with gynecological malignancies? Despite the paucity of evidence, it is undeniable that robot-assisted surgery has rapidly gained popularity in the gynecologic oncology community. The purpose of this review is to assess the current state of robot-assisted surgery in gynecologic oncology. To achieve this goal, a PubMed search was conducted using the keywords "robotic", "robotic surgery", "endometrial cancer", "cervical cancer", and "ovarian cancer". Publications in the English language were reviewed for inclusion in this review article.

To compare cancer staging surgeries performed via laparotomy, traditional laparoscopy, or robot-assisted surgery, certain objective parameters are often used to compare the three modalities. Most studies use similar objective measurements to compare these different modalities. The most common operative results reviewed include estimated blood loss (EBL), operative time, the number of lymph nodes resected, and the number of patients that were converted from laparoscopy and/or robot-assisted surgery to laparotomy. Postoperative outcomes included length of postoperative hospitalization, surgical complications, and blood transfusions.

\section{Endometrial cancer}

Endometrial cancer is the most common female genital tract malignancy in the USA. An estimated 54,870 cases of uterine cancer are expected to be diagnosed in 2015, as well as an estimated 10,170 deaths from the disease. From 2007 to 2011 , the incidence rate increased by $2.4 \%$ per year, while the death rate during the same time period increased by $1.9 \%$ per year. ${ }^{10}$

As per the International Federation of Gynecology and Obstetrics (FIGO), total extrafascial hysterectomy and bilateral salpingo-oophorectomy with pelvic and paraaortic lymph node dissection is the standard staging procedure for endometrial cancer. ${ }^{11}$ The surgical staging can be performed via laparotomy, laparoscopy, or robot-assisted surgery.

The 2009 landmark GOG LAP2 study demonstrated that the optimal surgical route for patients with endometrial cancer is laparoscopic surgical staging. This was the first randomized controlled trial to compare laparoscopy and laparotomy for the comprehensive surgical staging of uterine cancer. ${ }^{12}$ Patients with clinical stage I to IIA uterine cancer were randomly assigned to laparoscopy $(n=1,696)$ or laparotomy $(n=920)$ at a 2:1 ratio. The surgical procedures included hysterectomy, salpingo-oophorectomy, peritoneal cytology, and pelvic and paraaortic lymphadenectomy. The study found that complications were more common in the laparotomy arm compared to the laparoscopic arm (21\% vs $14 \%$, respectively; $P<0.001)$, even after controlling for patient age, race/ethnicity, body mass index (BMI), and performance status.

Specifically, complications such as ileus occurred significantly more often with laparotomy compared to laparoscopy (7\% vs 4\%, respectively; $P<0.004$ ) as did cardiac arrhythmia ( $2 \%$ vs $1 \%)$. Additionally, the proportion of patients 
requiring $>2$ days of hospitalization after surgery was significantly less in patients undergoing laparoscopy compared to laparotomy ( $52 \%$ vs $94 \%$, respectively; $P<0.0001$ ). This was true even after controlling for age, race/ethnicity, BMI, and performance status.

Paraaortic lymph node retrieval was documented in 97\% of patients undergoing laparotomy, while in the laparoscopic arm the retrieval rate was noted to be $94 \%(P=0.002)$. Additionally, both paraaortic and pelvic lymph nodes were retrieved in $96 \%$ of patients undergoing laparotomy and in $92 \%$ of those undergoing laparoscopy $(P<0.001)$. Peritoneal fluid or washings were examined cytologically in $90 \%$ of laparotomy patients and $96 \%$ of laparoscopic patients $(P=0.052)$. The proportion of participants randomly assigned to the laparotomy and laparoscopic arms and found to have an advanced surgical stage was not significantly different between the groups ( $17 \%$ vs $17 \%$, respectively; $P=0.851$ ). The conversion rate was $25.8 \%$. The reported reasons for conversion were poor exposure (56.7\%), cancer requiring laparotomy for adequate resection (15.9\%), excessive bleeding (11.3\%), equipment failure, and other causes.

A subsequent analysis of this study ${ }^{13}$ had a median follow-up time of 59 months for the 2,181 patients that were still alive. There were 309 recurrences (210 laparoscopy; 99 laparotomy) and 350 deaths (229 laparoscopy; 121 laparotomy). The estimated 3-year recurrence rate was similar for both groups ( $11.4 \%$ vs $10.2 \%$ for the laparoscopic and laparotomy group, respectively), with a difference of $1.14 \%$. The estimated 5 -year overall survival was almost identical in both arms at $89.8 \%$.

Despite the significant findings of the GOG LAP2 study, surgeons have been slow to utilize laparoscopy for the management of endometrial cancer. The impedance to its adaptation is likely the steep learning curve and non-ergonomic experience associated with traditional laparoscopy. Robot-assisted surgery, another form of minimally invasive surgery, may afford the same benefits of the laparoscopic approach while having a lower learning curve and providing a greater ergonomic experience for the surgeon. While endometrial cancer staging is the most common indication for the use of robot-assisted surgery, ${ }^{5}$ there are no large randomized controlled trials comparing robot-assisted surgery to laparotomy. However, several retrospective studies directly compare laparotomy, traditional laparoscopy, and robot-assisted surgical approaches for the management of endometrial cancer. ${ }^{7,8,14-16}$

Table 1 provides a summary of studies that investigate robot-assisted surgery in endometrial cancer.

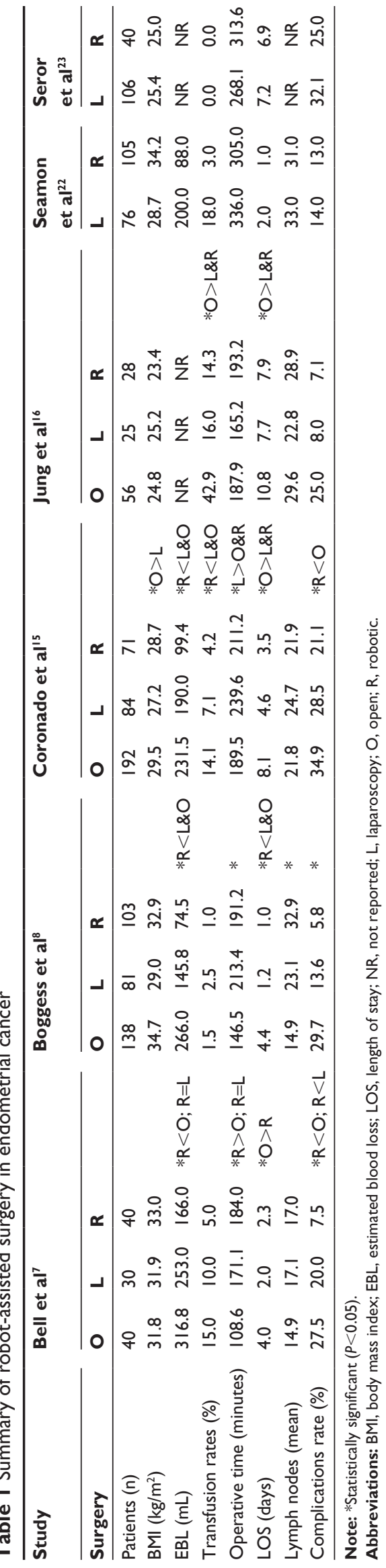


This table provides four studies that compare laparotomy with traditional laparoscopy and robot-assisted surgery for the surgical management of endometrial cancer. Additionally, two studies are depicted comparing traditional laparoscopy with robot-assisted surgery.

We reviewed six studies that compared laparotomy with traditional laparoscopy and robot-assisted surgery for the surgical management of endometrial cancer. Most of these studies did not differ with regard to age or BMI. ${ }^{8,14,16,17}$ However, in the Bell et al study, the total robot-assisted hysterectomy (TRH) group was younger than both the total abdominal hysterectomy (TAH) and total laparoscopic hysterectomy (TLH) groups. ${ }^{7}$ Additionally, in the Coronado et al study, patients undergoing TAH had a higher BMI than those undergoing TLH. ${ }^{15}$

A significant cause of morbidity associated with surgery is EBL, as this often affects healing and overall postoperative health, especially in oncological patients. All of the reviewed studies that recorded EBL demonstrated that it was less with TRH compared to TAH, which had the greatest EBL. ${ }^{7,8,14-17}$ Four of the six studies showed that patients undergoing robot-assisted surgery had the lowest percentage of patients receiving blood transfusions. ${ }^{7,8,15,16}$ Chiou et al did not record the numbers of patients requiring blood transfusion. ${ }^{14}$

The determination of lymph node involvement is necessary to optimally tailor adjuvant therapies and reduce local and distant recurrences, and is therefore extremely valuable in endometrial cancer staging. ${ }^{18}$ When comparing lymph node yield, Boggess et $\mathrm{al}^{8}$ found that the total number of lymph nodes retrieved was greater in TRH compared to TAH, while Bell et $\mathrm{al}^{7}$ and Chiou et $\mathrm{al}^{14}$ found no difference in the total number of nodes retrieved when the three modalities were compared. When specifically looking at pelvic lymph nodes retrieved, Boggess et $\mathrm{al}^{8}$ and Jung et $\mathrm{al}^{16}$ found that more pelvic lymph nodes were obtained in TRH versus TLH, whereas both Coronado et $\mathrm{al}^{15}$ and Corrado et $\mathrm{al}^{17}$ found no difference in retrieval. A greater number of paraaortic lymph nodes were retrieved in the TRH group compared to the TLH group in the Boggess et $\mathrm{al}^{8}$ study, but Jung et $\mathrm{al}^{16}$ and Coronado et $\mathrm{al}^{15}$ found no difference in lymph node retrieval between the three groups. Corrado et al, however, found that a greater number of paraaortic lymph nodes were retrieved in TAH compared to TRH. ${ }^{17}$

Prolonged post-procedure hospitalization may be associated with morbidity related to nosocomial infection and thromboembolism. Additionally, it is directly related to the overall cost and financial burden to the patient and the health care system. All six studies reviewed ${ }^{7,8,14-17}$ found that the length of post-procedure hospitalization was less in both TRH and TLH when compared to TAH. Furthermore, four of the six studies found that the length of stay was the least with TRH. ${ }^{8,14,15,17}$

It is important to examine the operative time among the three modalities since it is often used as a quality marker. Prolonged operative time is associated with increased cost and potential morbidity to patients. Bell et $\mathrm{al}^{7}$ and Boggess et $\mathrm{al}^{8}$ found that the length of surgery was greater with TRH than TAH. Jung et al found no significant difference in length of surgery between the three routes of surgery. ${ }^{16}$ Coronado et al found that TRH was faster than TLH and found no difference in length of surgery between TAH and TRH. ${ }^{15}$ Interestingly, Chiou et $\mathrm{al}^{14}$ and Corrado et $\mathrm{al}^{17}$ found that both TRH and TLH were faster than TAH. Perhaps Bell et $\mathrm{al}^{7}$ and Boggess et $\mathrm{al}^{8}$ did not find robot-assisted surgery to be faster since both of these studies were published in 2008 and their robotic experience may have been limited compared to studies that were published several years later.

Overall complications were lower in TRH compared to TAH in four of the reviewed studies..$^{8,15-17}$ Chiou et al ${ }^{14}$ found no significant difference in overall complications between the three groups, while Boggess et $\mathrm{al}^{8}$ did not specifically record the overall complications. With regard to intraoperative complications, the highest percentage of intraoperative complications was found to be associated with either $\mathrm{TLH}^{8,15}$ or TAH. ${ }^{8,16,17}$ Postoperative complications were less with TRH compared to TAH in four of these studies. ${ }^{7,8,15,17}$ Specifically, there was a lower incidence of ileus, wound dehiscence or wound separation, cellulitis, and wound infection in the TRH groups. ${ }^{7,8,15}$ Conversion rate was found to be highest in the laparoscopic group in several studies. ${ }^{8,15,17}$

Obesity is a well-known risk factor for developing endometrial cancer, and therefore many of these patients have a BMI $>30$. Two studies compared TRH versus TAH specifically in obese patients. ${ }^{19,20}$ Neither of the studies found a difference in the total number of lymph nodes retrieved. Seamon et al further investigated the number of nodes recovered with regard to the anatomic location and found that TRH yielded a significantly higher number of left peraaortic nodes compared to TAH. ${ }^{19}$ Both studies demonstrated a significantly greater EBL, percentage of patients requiring blood transfusions, and wound problems in patients undergoing TAH. Operative time (minutes) was greater in TRH compared to TAH (Seamon et al: ${ }^{19} 228$ vs 143, $P<0.001$; Subramaniam et al: ${ }^{20} 246.2$ vs 138.2, $P<0.001)$. Conversion rates in these two studies were similar at $15.6 \%$ and $11 \%$.

Four studies directly compared TLH to TRH in the surgical management of endometrial cancer. ${ }^{21-24}$ Three of these 
studies found no differences in the number of paraaortic or pelvic lymph nodes retrieved. However, Frey et al found that robot-assisted surgery was associated with a greater lymph node harvest with no increase in operative time or perioperative complications. ${ }^{24}$ Seamon et al found that the EBL, the number of patients requiring blood transfusion, and the length of stay after procedure were less in TRH versus TLH, but did not demonstrate a difference in complications. ${ }^{22}$ Seror et al found no difference between TLH and TRH in the number of patients requiring blood transfusions, the length of stay after procedure, or complications. ${ }^{23}$ In terms of conversion rate, Cardenas-Goicoechea et $\mathrm{al}^{21}$ found no difference in conversion rate, while Seamon et $\mathrm{al}^{22}$ found that conversion occurred less often in TRH versus TLH $(12 \%$ vs $26 \%, P=0.017)$. Furthermore, Seamon et al found that the odds of having a conversion to laparotomy are $80 \%$ less for robot-assisted surgery compared to traditional laparoscopy. ${ }^{22}$ With regard to operating room time, Seaman et $\mathrm{al}^{13}$ found that time in the operating room was shorter for TRH than TLH, while Seror et $\mathrm{al}^{23}$ found the opposite to be true. This difference in operative time may be attributed to the surgeons' experience.

Limited data exist on long-term oncological outcomes in regard to robot-assisted surgery in the management of endometrial caner. However, the LAP2 study demonstrated that the potential for increased risk of cancer recurrence with laparoscopy versus laparotomy was small, and the estimated 5-year overall survival was almost identical. Although there is not yet a large randomized trial investigating survival outcomes with robot-assisted surgery, some information may be gleamed from retrospective studies. Several retrospective studies comparing robot-assisted surgery with laparoscopy and laparotomy for the management of endometrial cancer found no differences in disease-free survival and overall survival. ${ }^{14,15,17}$ Cardenas-Goicoechea et al compared the survival analysis of robot-assisted versus traditional laparoscopic surgical staging for endometrial cancer and found no difference in disease-free survival or overall survival between robot-assisted or laparoscopic staging. ${ }^{21}$

In summary, robot-assisted surgery utilized in the management of endometrial cancer is associated with lower EBL, shorter length of hospital stay, and fewer complications compared to laparotomy. It also appears that robot-assisted surgery is able to deliver at least the same, if not greater, yield of lymph nodes compared to laparotomy. Studies also demonstrate that robot-assisted surgery is superior to laparotomy in the obese population. While data is limited, it appears that disease-free survival or overall survival between TAH, TLH, and TRH is small or nonexistent. The benefits of minimally invasive surgery (TLH or TRH) over laparotomy are obvious, whereas the advantages of TLH versus TRH are less clear and require further study.

\section{Costs analysis}

Robot-assisted surgery has increased in popularity among gynecologic oncologists for the surgical management of endometrial cancer, but at what cost? Understandably, one of the largest concerns expressed regarding the adoption of robotic technology is its high cost. Currently, the only available robot-assisted surgical system, the da Vinci surgical system (Intuitive Surgical, Sunnyvale, CA, USA), has a fixed cost between $\$ 1.5$ million and $\$ 1.75$ million. ${ }^{5}$ In addition to the fixed cost of purchasing the platform, one must also take into account variable costs such as operating room supplies, drapes, and operating room time.

Several studies have compared the costs of robot-assisted surgery to other modalities for the surgical management of endometrial cancer. One of the first studies to make this comparison analyzed the direct and indirect costs of 110 patients who underwent surgical staging via laparotomy, traditional laparoscopy, and robot-assisted surgery. This study found that the average cost for hysterectomy with staging via laparotomy was the most expensive $(\$ 12,943.60)$, followed by robot-assisted surgery $(\$ 8,212.00)$, and finally traditional laparoscopy $(\$ 7,569.80)$. The differences in costs between laparotomy and robot-assisted surgery were statistically significant $(P=0.0001)$, while the difference in costs between traditional laparoscopy and robot-assisted surgery were not statistically significant $(P=0.06)$. The authors concluded laparotomy was the most expensive modality, likely associated with the increased length of stay and the increased number of days needed to return to normal activity. ${ }^{7}$

Another study compared the surgical costs for endometrial cancer staging for 33 patients undergoing either traditional laparoscopy or robot-assisted surgery. This group found that mean operative costs were higher for robot-assisted procedures $(\$ 3,323$ vs $\$ 2,029 ; P<0.001)$, in part because of longer operating room time. However, the most significant contributor to the cost difference was due to disposable instrumentation $(\$ 1,755$ vs $\$ 672 ; P<0.001)$. Additionally, total hospital costs were higher for robot-assisted surgeries ( $\$ 5,084$ vs $\$ 3,615 ; P=0.002)$, most of this difference being attributed to overall operating room costs. ${ }^{25}$

A third study utilized the Perspective database to identify women who underwent a hysterectomy by either traditional laparoscopy or robot-assisted surgery for both benign indications and endometrial cancer. This database captures comprehensive 
billing data of all hospital admissions from $>500$ acute care facilities from throughout the USA. Between 2006 and 2012, 10,906 patients were identified that underwent hysterectomy for endometrial cancer. The unadjusted median cost of robotassisted hysterectomy for endometrial cancer was $\$ 9,691$ compared with $\$ 8,237$ for traditional laparoscopy $(P<0.001)$. Median fixed costs were $\$ 4,543$ for robot-assisted hysterectomy compared with $\$ 3,790$ for traditional laparoscopy, whereas variable costs were $\$ 5,065$ and $\$ 4,215$ for the two procedures, respectively. This study also examined the difference in cost between traditional laparoscopy and robot-assisted surgery based on the number of cases performed at each hospital and the number of cases performed by each surgeon. This study found that the difference in costs between the two modalities decreased substantially as the number of cases performed at hospitals and by individual surgeons increased. For example, at hospitals that performed fewer than five previous cases, robot-assisted surgery was $\$ 2,471$ more expensive than traditional laparoscopy, whereas at hospitals that performed $>50$ cases the cost difference was $\$ 924$. Among surgeons, robot-assisted surgery was $\$ 1,761$ more expensive for those who had performed fewer than five cases, whereas the difference in costs declined to $\$ 688$ for those that performed $>50$ procedures. $^{26}$

Minimally invasive procedures are less costly than laparotomy when taking into account the length of stay and recovery times. ${ }^{5}$ Robot-assisted surgery appears to be more expensive than traditional laparoscopy largely due to increased operating room time and the cost of disposable instrumentation. As gynecologic oncologists continue to utilize robot-assisted surgery for the management of endometrial cancer, surgical volume and experience will increase, leading to a decrease in operating room time and therefore a further decrease in the cost difference between traditional laparoscopy and robotassisted surgery. Additionally, more companies will likely introduce competition into the market, which may drive down the associated costs of disposable instrumentation.

\section{Cervical cancer}

Cervical cancer is the second most common indication for the use of robot-assisted surgery. ${ }^{5}$ An estimated 12,900 cases of invasive cervical cancer are expected to be diagnosed in the USA in 2015, along with 4,100 deaths. Five- and 10-year relative survival rates for cervical cancer patients are $68 \%$ and $64 \%$, respectively. Almost half of patients (47\%) are diagnosed when the cancer is localized, for which the 5-year survival is $91 \%$. Five-year survival rates for regional and distant stage disease are $57 \%$ and $16 \%$, respectively. ${ }^{10}$

\section{Radical hysterectomy}

The standard surgical management for early-stage cervical cancer (IA2-IB1, 1B2, IIA) is abdominal radical hysterectomy with bilateral pelvic lymphadenectomy. ${ }^{27}$ Radical hysterectomy is a complicated and arduous surgery associated with significant complications, morbidity, and a long recovery. Applying minimally invasive techniques to reduce the comorbidities and length of recovery is beneficial to both the patients and the health care system. However, performing this surgery via traditional laparoscopy requires extensive training and is associated with a steep learning curve. The implementation of robot-assisted radical hysterectomy has allowed patients to reap the benefits of minimally invasive surgery while undergoing radical hysterectomy.

Since 2005, when the da Vinci surgical system (Intuitive Surgical) was first approved for gynecological surgery, there have been several studies investigating its use for radical hysterectomy in patients with cervical cancer. Comparing robot-assisted type III radical hysterectomy with pelvic lymphadenectomy to open radical hysterectomy, Boggess et al reported a case-control study on the treatment of patients with cervical cancer staged $1 \mathrm{~B} 1,1 \mathrm{~B} 2$, and $1 \mathrm{~A} 2 .{ }^{28}$ In this study, 51 patients underwent robot-assisted radical hysterectomy and were compared to 49 open radical hysterectomies that were performed prior to the implementation of robotassisted surgery at their facility. The incidence of patients with prior abdominal surgery was $51 \%$ in the robot-assisted cohort and only $18.4 \%$ in the laparotomy cohort $(P=0.04)$. Significant findings in the robot-assisted cohort included shorter operating times (210 minutes vs 248 minutes), shorter length of hospital stay (1 day vs 3.2 days), less EBL (96.5 mL vs $416.8 \mathrm{~mL}$ ), and a greater pelvic lymph node retrieval (33.8 vs 23.3 nodes). The incidence of blood transfusions among the laparotomy cohort was $8 \%$ compared with zero in the robot-assisted cohort. Of note, the average robot-assisted operative time significantly improved after the first 12 cases (243.4 minutes in the first 12 cases vs 193.3 minutes in the last 12 cases). In another study, Persson et al also demonstrated that the operative time for robot-assisted radical hysterectomy decreased as the number of completed procedures increased (176 minutes after nine cases vs 132 minutes after 26 cases). ${ }^{29}$ The complication rate in the robot-assisted cohort in the Boggess et al study was $7.8 \%$ compared to $16.3 \%$ in the laparotomy cohort; however, this was not found to be statistically significant. ${ }^{8,28}$

Other studies have also demonstrated that robot-assisted radical hysterectomy is associated with a lower EBL and a 
shorter length of hospitalization when compared to open radical hysterectomy. ${ }^{27,28,30-33}$ In addition to having a shorter length of stay, Asciutto et al also found that the robot-assisted group had a shorter time to the resumption of normal activities of daily living. ${ }^{30}$ Additionally, most studies have reconfirmed that robot-assisted surgery, when compared to laparotomy, has an equivalent or greater lymph node retrieval rate while still maintaining a lower postoperative complication rate. ${ }^{31-33}$

Magrina et al also reported on the utility of robot-assisted radical hysterectomy in the surgical management of cervical cancer. ${ }^{33}$ This prospective analysis compared the perioperative results of robot-assisted radical hysterectomy to those performed by either traditional laparoscopy or laparotomy. The three groups were matched by age, BMI, site and type of malignancy, FIGO staging, and type of radical hysterectomy. The mean operating time was significantly longer for the laparoscopic group when compared to either the robot-assisted or laparotomy group (220.4 minutes vs 189.6 minutes vs 166.8 minutes, respectively). Patients undergoing robotassisted surgery and laparoscopy, when compared to laparotomy, had a significantly lower mean blood loss (133.1 mL vs $208.4 \mathrm{~mL}$ vs $443.6 \mathrm{~mL}$, respectively), mean rate of blood loss ( $0.7 \mathrm{~mL} / \mathrm{min}$ vs $0.9 \mathrm{~mL} / \mathrm{min}$ vs $2.6 \mathrm{~mL} / \mathrm{min}$, respectively), and mean length of hospitalization ( 1.7 days vs 2.4 days vs 3.6 days, respectively). However, lymph node retrieval and intraoperative and postoperative complication rates were similar among the three groups.

Several studies reviewed in the literature directly compared traditional laparoscopy to robot-assisted surgery in the performance of radical hysterectomy. Most of these studies found no difference between the two modalities with regard to $\mathrm{EBL}^{31,34}$ or length of hospitalization. ${ }^{31,34,35}$ However, there have been some exceptions that demonstrate that robot-assisted surgery may have a slight advantage. For example, Diaz-Feijoo et $\mathrm{al}^{35}$ found robot-assisted radical hysterectomies to be associated with a lower EBL, while Soliman et $\mathrm{al}^{36}$ found that the length of stay was significantly shorter in the robot-assisted group. Soliman et $\mathrm{al}^{36}$ also evaluated the analgesic and antiemetic requirements after minimally invasive surgery and discovered that total intravenous opioids administered postoperatively were significantly higher in the laparoscopic group when compared to the robot-assisted group ( $26.7 \mathrm{mg}$ vs $10.7 \mathrm{mg}, P=0.001$ ). Additionally, some studies have demonstrated that lymph node retrieval may be greater with robot-assisted surgery. ${ }^{31,35}$ Robot-assisted surgery is also associated with a shorter time to resumption of normal activities postoperatively. ${ }^{30}$
Overall, robot-assisted radical hysterectomy appears to be equivalent or superior to open radical hysterectomy in terms of EBL, length of hospitalization, lymph node retrieval, and postoperative complications. Additionally, it seems that perioperative outcomes are similar when radical hysterectomy is performed by either robot-assisted or traditional laparoscopic surgery. As some suggest, more studies are needed to conclude whether robot-assisted surgery may have an advantage over traditional laparoscopy.

Table 2 provides several studies that evaluated robotassisted surgery utilization in radical hysterectomy.

\section{Paraaortic lymphadenectomy}

Understanding the extent of disease is an important aspect in the management of cervical cancer, as it helps to guide treatment. Lymphadenectomy provides definitive information with regard to the involvement of the lymph nodes, not only in the pelvis but also along the chain of lymph nodes around the aorta. ${ }^{37}$ This is important since imaging techniques are not $100 \%$ sensitive in detecting paraaortic nodal metastasis. Moreover, the implementation of appropriate treatment following identification and removal of histologically positive paraaortic nodes results in a respectable 5 -year survival of $50 \%$ as compared to a dismal or no survival if undetected and untreated. ${ }^{35}$ Additionally, involvement of paraaortic lymph nodes may require extension of the radiation field to the lower abdomen to fully cover the sites of disease. ${ }^{6}$

Both an extraperitoneal and a transperitoneal approach to paraaortic lymphadenectomy have been described in the literature. The extraperitoneal laparoscopic approach has been shown to have some advantages over the transperitoneal route due to decreased risk of bowel injury, avoidance of preexisting abdominal adhesions, and reduced adhesion formation. ${ }^{35}$ Diaz-Feijoo et al compared robot-assisted extraperitoneal paraaortic lymphadenectomy to the traditional laparoscopic approach and found no difference between robot-assisted and traditional laparoscopy in terms of operating time, hospital stay, and postoperative complications. ${ }^{35}$ A statistically significant difference was observed for lower blood loss and higher number of nodes removed in the robot-assisted group (20 mL, 17 nodes) compared to the traditional laparoscopic group ( $90 \mathrm{~mL}, 14$ nodes). However, the clinical significance of these findings is likely minimal to nonexistent. It is important to note that this series had a small number of participants ( $n=83$ traditional laparoscopy vs $n=17$ robot-assisted) and the BMI between the two groups was statistically different. However, other studies in the literature demonstrate similar 


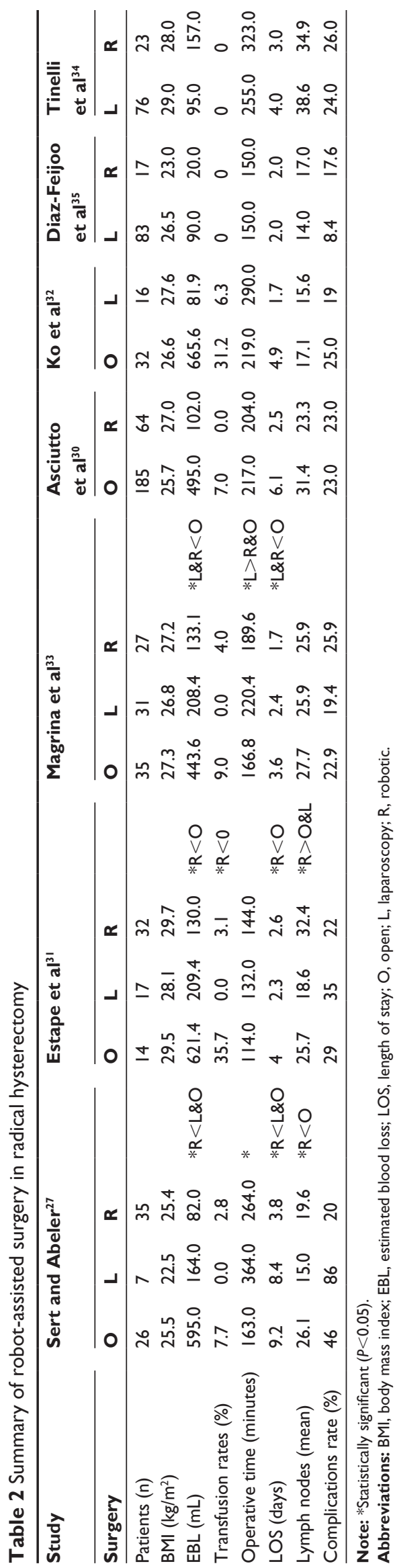

perioperative data. ${ }^{38,39}$ The literature also contains several studies that explain the logistics and demonstrate the feasibility of performing robot-assisted transperitoneal paraaortic lymphadenectomy. ${ }^{40-42}$

\section{Trachelectomy}

It is estimated that $>40 \%$ of cervical cancer cases diagnosed in the USA occur in women of reproductive age ${ }^{43}$ Well-selected, reproductive-aged women diagnosed with cervical cancer with a tumor size of $<2 \mathrm{~cm}$ may be candidates for radical trachelectomy and lymphadenectomy. This procedure can be performed vaginally, abdominally, laparoscopically, or robotically. Robotassisted trachelectomy has been reported in the literature. ${ }^{43-51}$ It offers advantages over other routes of surgery that aid in the ability to perform a meticulous dissection with extraordinary dexterity. Some of these advantages include increased depth perception, three-dimensional stereoscopic vision, wristed instruments with seven degrees of freedom allowing ease of suturing, and tremor-canceling software.

Geisler et al reported the first robot-assisted radical trachelectomy in $2008 .{ }^{47}$ Since that time, other studies have evaluated its utilization for performing a trachelectomy. Specifically, Persson et al assessed the accuracy and reproducibility of robot-assisted, fertility-sparing radical trachelectomy and compared it to vaginal trachelectomy. ${ }^{50}$ This group concluded that robot-assisted trachelectomy is as equally reproducible and accurate as the vaginal route in terms of cervical length and that robot-assisted trachelectomy with cerclage placement resulted in a significantly more precise placement of the cervical cerclage. In this same study, all cases of cerclage rejection and/or cervical stenosis were diagnosed in patients in the vaginal cohort. ${ }^{50}$ In another study, Nick et al compared open versus robot-assisted surgical approaches for trachelectomy and found that those in the robot-assisted group had less blood loss and a decreased length of postoperative stay. ${ }^{49}$ Importantly, there was a higher rate of conversion to hysterectomy in the robot-assisted cohort, but rates of serious morbidities between the two cohorts were comparable..$^{49}$ Robot-assisted trachelectomy with cerclage placement has proven to be both feasible and reproducible, and several authors have described this surgical technique..$^{44,46,51}$ Performing a robot-assisted trachelectomy may allow more surgeons to perform this surgery minimally invasively, thereby affording patients the associated benefits.

\section{Parametrectomy}

The outcomes of suboptimal management of cervical cancer, discovered on pathology after a simple hysterectomy, were 
significantly compromised in the absence of additional treatments with an approximate recurrence rate of $60 \%$ and an estimated 5-year overall survival of $<50 \%{ }^{52}$ Therapeutic options include pelvic radiation with chemosensitization or radical parametrectomy. The latter is preferred when preservation of vaginal and ovarian function is desired.

Robot-assisted radical parametrectomy and pelvic lymphadenectomy have been reported to be safe and feasible. ${ }^{52,53}$ Vitobello et al reported their experiences and performed a literature review, which found that robot-assisted surgery represents an effective alternative to accomplish radical parametrectomy, thereby avoiding radiotherapy in $>80 \%$ of cases. ${ }^{52}$ Magrina and Magtibay described a safe and feasible technique of a nerve-sparing radical parametrectomy that resulted in a decreased proportion of patients with bladder and rectal dysfunction as compared to conventional radical hysterectomy. ${ }^{54}$

\section{Exenteration}

Exenteration is an extensive operation for central pelvic tumor recurrence. Three types of pelvic exenteration have been described. Anterior exenteration is the removal of the bladder, uterus, cervix, and all or part of the vagina. Posterior pelvic exenteration is the removal of the anus and rectum and resection of the uterus, cervix, and all or part of the vagina. Total exenteration is the combination of the two, thereby removing all the pelvic contents. Prior to undergoing an exenteration, it is necessary to thoroughly evaluate for any evidence of disease outside the pelvis, as this would be a contraindication. Severe postoperative and intraoperative complications can occur with this procedure, and perioperative mortalities as high as $10 \%-20 \%$ have been reported. Major risks include infection and bowel obstruction. ${ }^{55}$

The benefits of performing an exenteration with minimal invasiveness are intuitive. The da Vinci robotic system affords the surgeon the necessary dexterity and fine motor movements to successfully dissect into the confines of the narrow pelvic floor while also allowing the fine suturing that is required. For these reasons, Lim attempted and successfully completed the first robot-assisted total pelvic exenteration with an ileal loop urinary diversion and an end colostomy in $2009 .{ }^{56}$ Total operative time was 375 minutes, no intraoperative or postoperative complications were reported, and the EBL was $375 \mathrm{~mL}$. Impressively, the patient was discharged home on postoperative day 10 after colostomy output was documented. Since then, there have been other successful reports of robot-assisted exenterations. ${ }^{57,58}$ Iavazzo and Gkegkes's review article ${ }^{59}$ describing robot-assisted surgery in the performance of pelvic exenterations included four studies with a total of eight patients. This review article concluded that the robot-assisted surgery might be an alternative to laparotomy.

\section{Cost analysis}

As previously discussed with endometrial cancer, the perceived cost of robot-assisted surgery is often an argument used against its widespread implementation. Wright et al identified women recorded in the Perspective database with cervical cancer that underwent radical hysterectomy (abdominal, laparoscopic, robotic) from 2006 to 2010. Of the 1,894 patients included, 1,610 (85\%) underwent abdominal, 217 (11.5\%) underwent laparoscopic, and 67 (3.5\%) underwent robot-assisted radical hysterectomy, and were analyzed. ${ }^{60}$ They found median costs were $\$ 9,618$ for abdominal, \$11,774 for laparoscopic, and \$10,176 for robotassisted radical hysterectomy $(P<0.0001)$. In a multivariable model, the cost of laparoscopic radical hysterectomy $(95 \%$ confidence interval [CI], \$457-\$1,576) was associated with $\$ 1,016$ in higher costs compared to abdominal hysterectomy, while the cost of robot-assisted radical hysterectomy $(\$ 789$; 95\% CI, \$248-\$1,827) was not statistically different from that of an abdominal procedure. ${ }^{26}$ Reynisson et al had similar findings and found that, given 400 robot-assisted operations annually and after a substantial implementation period, it is feasible to perform robot-assisted radical hysterectomies at an equal hospital cost compared with laparotomy. ${ }^{61}$

\section{Ovarian cancer}

An estimated 21,290 new cases of ovarian cancer are expected in the USA in 2015, while an estimated 14,180 deaths are expected. Ovarian cancer accounts for $5 \%$ of cancer deaths among women, causing more deaths than any other gynecologic cancer. Overall, the 5- and 10-year relative survival rates for ovarian cancer patients are $45 \%$ and $35 \%$, respectively. However, survival varies substantially by age; women younger than 65 are twice as likely to survive 5 years as women 65 and older ( $58 \%$ vs $27 \%$ ). Overall, only $15 \%$ of cases are diagnosed at an early stage, for which the 5-year survival is rate $92 \%$. The majority of cases $(61 \%)$ are diagnosed at a late stage, for which the 5-year survival rate is $27 \% .^{10}$

There is less literature on robot-assisted surgery in ovarian cancer compared to its utilization in both endometrial and cervical cancers. However, there are a few small retrospective studies evaluating the robot's utility in the management of ovarian cancer. ${ }^{62-64}$ It is essential to note that these studies are very small and therefore require great skepticism. 
In a retrospective case-control analysis of perioperative outcomes and survival, Magrina et al examined patients undergoing primary surgical treatment of epithelial cancer by robot-assisted surgery, traditional laparoscopy, or exploratory laparotomy. ${ }^{64}$ The robot-assisted and traditional laparoscopic groups only had 25 and 27 subjects, respectively, while the laparotomy group consisted of 119 subjects. Patients in each surgical group were further classified into three groups according to the extent of debulking and the type and number of major surgical procedures performed at the time of the initial surgery. Significant findings included the operating time, which was higher for robot-assisted surgery (315 minutes) compared to traditional laparoscopy (254 minutes) or laparotomy (261 minutes). Furthermore, EBL was significantly higher in the laparotomy group $(1,308 \mathrm{~mL})$ compared with either laparoscopy $(267 \mathrm{~mL})$ or robot-assisted surgery (164 mL). Length of stay was also significantly higher in the laparotomy group (9 days) compared to laparoscopy (3 days) or robot-assisted surgery (4 days). Finally, postoperative complications were greatest in both the laparotomy $(33 \%)$ and robot-assisted (24\%) groups compared to laparoscopy (24\%). Intraoperative complications and lymph node yields were similar among the three groups.

Magrina et al also found that progression-free survival was significantly higher for the robot-assisted and laparoscopic groups compared to the laparotomy group. ${ }^{64}$ This finding raises doubt, as several other contributing factors may account for this difference besides the modality of surgical intervention. First, it is unlikely that this study would have been able to detect a progression-free survival difference between groups with such small numbers. Second, the groups were not randomized and there were notable differences that may account for their findings. For example, patients with more disseminated disease, and therefore disease, more likely to recur at an earlier date were selected to undergo laparotomy. Additionally, the robot-assisted and laparoscopic groups had a higher overall proportion of patients (48.0\% and $40.7 \%$ ) receiving neoadjuvant or adjuvant IP chemotherapy as compared to the laparotomy group (36.5\%), which by itself may account for a greater progression-free survival. Also, FIGO staging III-IV was allocated unevenly: $60 \%, 75 \%$, and $87 \%$ for robotics, laparoscopy, and laparotomy, respectively. Finally, complete debulking was achieved in $84 \%$ of patients in the robot-assisted group, 93\% in the laparoscopic group, and only $56 \%$ in the laparotomy group.

As expected, no difference was noted in overall survival among the three groups. Magrina et al concluded that robotassisted surgery and traditional laparoscopy appear preferable to laparotomy for the surgical treatment of ovarian cancer patients requiring primary tumor excision alone or with one additional major procedure. ${ }^{64}$ Additionally, laparotomy remains preferable for patients requiring primary tumor excision and two or more major procedures. ${ }^{64}$

In another small study, Feuer et al evaluated the feasibility and efficacy of robot-assisted surgery in the management of epithelial ovarian cancer compared to laparotomy. ${ }^{63}$ This was a retrospective review with only 99 subjects: 63 completed robotically and 26 by laparotomy. Cases were compared that were similar for age, uterine weight, and BMI. However, a history of prior abdominal surgery was more common in the laparotomy group ( $96.2 \%$ vs $76.2 \%, P=0.0257)$. Robotassisted operative times were significantly longer (138.6 minutes vs 95.2 minutes), while EBL (94.9 mL vs $385.4 \mathrm{~mL}$ ) and length of stay (2.3 days vs 6.2 days) were significantly lower in the robot-assisted group. Major complication rates (16\% vs $23 \%, P=0.4208)$ and lymphadenectomy yields (13 vs 11 nodes $P=0.2310$ ) were similar in both the robot-assisted and laparotomy groups, respectively. At 1 year, the survival ( $97 \%$ vs $90 \%, P=0.2501)$ and no evidence of disease rates $(80.6 \%$ vs $85 \%, P=0.6773)$ were equivalent in the robotassisted and laparotomy groups, respectively.

We are very critical of this study's finding that recovery time was shorter in the robot-assisted group and that more patients were discharge after 1 day of hospitalization. The most likely reason for these findings is that nearly $38 \%$ of patients in the laparotomy group underwent bowel resections, whereas no patients in the robot-assisted group experienced a bowel resection. Additionally, a significantly greater number of patients in the robot-assisted group received neoadjuvant chemotherapy ( $52 \%$ vs $15 \%, P=0.0013)$, thereby likely contributing to a less arduous surgical procedure. Perhaps, it is neoadjuvant chemotherapy that makes the surgery easier, not the utilization of robot-assisted surgery. Overall, Feuer et al concluded that an experienced robotic oncologic surgeon may be able to convert up to $80 \%$ of advanced ovarian cases to a minimally invasive surgical approach without compromising on safety or efficacy of treatment. ${ }^{63}$

In a third study that we reviewed, Chen et al evaluated the feasibility of robot-assisted surgery in the surgical management of ovarian cancer and compared its surgical outcomes with those of traditional laparoscopy and laparotomy. ${ }^{62}$ There were 138 subjects incorporated into this study (73 laparotomy, 44 robot-assisted, and 21 traditional laparoscopy). There were no significant differences found between the groups with regard to age, BMI, disease stage, histological type, optimal debulking rate, and cases with positive lymph nodes. 
The mean operation time was significantly less in the robot-assisted (176.8 minutes) and laparoscopic (232.3 minutes) groups compared with the laparotomy group (287.2 minutes) $(P=0.001)$. The mean EBL during the operations was also significantly decreased in the robot-assisted $(96.9 \mathrm{~mL})$ and laparoscopic $(326.2 \mathrm{~mL})$ groups compared with the laparotomy group (848.6) $(P<0.001)$, as were the transfusion rates $(0 \%$ robot-assisted, $14.3 \%$ laparoscopic, $46.6 \%$ laparotomy, $P<0.001)$. The robot-assisted group (2.7) demonstrated a significantly lower postoperative mean pain score than did the laparoscopic (4.6) and laparotomy (5.2) groups $(P<0.001)$. Additionally, the 24 -hour postoperative mean pain scores of the robot-assisted (2.0) and laparoscopic (3.2) groups were significantly lower than that of the laparotomy group (4.5) $(P<0.001)$. The mean times to resume full diets after surgery were significantly lower with robot-assisted (1.9 days) and laparoscopic (2.1 days) surgery compared to laparotomy (3.7 days) $(P=0.001)$. The mean duration of hospital stay was shorter in the robot-assisted (3.5 days) and laparoscopic (5.5 days) groups, as compared to the laparotomy (9.7 days) group $(P<0.001)$. The overall complication rates were not statistically significant $(P=0.13)$ between the robot-assisted (2.3\%), laparoscopic (4.8\%), and laparotomy (12.4\%) approaches. Given the small number of subjects in this study, it is of little surprise that no significant differences were observed with regard to disease-free survival or overall survival.

While this study found a statically significant difference in operating time among the three groups, this finding deserves more attention. This study considered the operative time for laparotomy to be skin to skin, while for both robot-assisted and laparoscopic surgery it was calculated as skin incision to closure minus the docking time. We find this to be undesirable. Docking should be incorporated into operative time, as it is clinically relevant since patients are still being subjected to the associated morbidities of anesthesia and operating room costs are still accruing while docking. With regard to the pain score findings, this study does not mention whether the patients or data collectors were blinded, nor does the study mention exclusion criteria such as chronic pelvic pain or other pain-associated conditions.

In summary, studies evaluating robot-assisted surgery in the management of ovarian cancer contain a small number of subjects and have several flaws. It does appear that robot-assisted surgery is feasible in the surgical management of ovarian cancer. These limited studies consistently demonstrate that the benefits of robot-assisted surgery, as observed in its utilization in endometrial and cervical cancer (decreased
EBL, shorter length of stay, etc), are also evident in its utilization in the management of ovarian cancer. However, the question of whether there is a survival difference when robot-assisted surgery is implemented in management of ovarian cancer is nowhere near answered.

\section{Looking forward Sentinel lymph node mapping}

The use of sentinel lymph node (SLN) mapping in the management of melanoma, breast cancer, and vulvar cancer has become the standard of care. One goal of the utilization of SLN mapping is to minimize both the rate of unnecessary lymphadenectomy in low-risk women, as well as the risk of understaging and undertreatment. ${ }^{65}$ SLN mapping for uterine and cervical malignancies has been gaining acceptance and may offer a potential alternative to full lymph node dissection in the future. ${ }^{66}$ Two dyes typically used in SLN mapping are isosulfan blue (ISB) and indocyanine green (ICG). ISB stains the surrounding tissue and relies on the surgeon's ability to visualize blue lymph nodes and lymph channels while differentiating them from surrounding tissue. Recently, it has been reported that the use of ICG in robot-assisted surgery is feasible, safe, time-efficient and a reliable method for lymphatic mapping in early-stage cervical and endometrial cancer. ${ }^{67}$

Near-infrared (NIR) fluorescence imaging provides the technology to perform SLN biopsy in a minimally invasive manner for both endometrial and cervical cancers utilizing ICG. ICG is the only FDA-approved fluorophobe in use for many years, but it has not yet been approved specifically for SLN mapping in the USA. ${ }^{66}$ This fluorescent dye appears green when excited by light in the NIR, is readably visible through visceral fat, ${ }^{65}$ and does not stain the surrounding tissues.

There are several NIR imaging capabilities available today. The FIREFLY is an integrated system used in conjunction with the da Vinci robot. It received FDA approval for use with the da Vinci ${ }^{\circledR} \mathrm{Xi}^{\mathrm{TM}}$ Surgical System (Intuitive Surgical) in August 2014. Additionally, NIR is available for laparoscopic and open approaches (PINPOINT and SPY Elite [Novadaq Technologies, Bonita Springs, FL, USA]). The fluorescence imaging equipment includes a fluorescence-capable illuminator, a camera head, and an endoscope, allowing rapid conversion from the normal robotic view to that which shows the location of ICG. ${ }^{68}$

It has been reported that fluorescence imaging with ICG detects bilateral SLN and SLN metastasis more often than does ISB ${ }^{69}$ and that it may be superior to colorimetric imaging with ISB in women undergoing SLN mapping for endometrial cancer ${ }^{65,69}$ While assessing the detection rate of SLNs using 
ICG and NIR imaging with the robotic platform for uterine and cervical malignancies, Jewel et al found that its use has a high bilateral SLN detection rate and appears favorable to using the blue dye alone and/or other modalities. ${ }^{66}$ Additionally, the combined use of ICG and ISB appears unnecessary. ${ }^{66}$

As mentioned throughout this review article, robot-assisted surgery has several advantages over traditional laparoscopy, which include high definition three-dimensional field of vision, instruments with wrist-like range of motion, tremor filtration, and better ergonomics. The summation of these advantages contributes to a less steep learning curve compared to traditional laparoscopy, thereby affording a greater number of surgeons to identify SLNs through minimally invasive means.

\section{Single-site robot-assisted surgery}

Conventional wisdom implies that less is better in terms of surgery and morbidity. It has been demonstrated that minimally invasive surgery is advantageous compared to laparotomy, as discussed throughout this review article. A small single incision would eliminate injuries related to lateral port placement (inferior epigastric vessel injury), decrease the number of incisions, and lower the probability of port-site herniation and infection while also improving cosmesis. The next frontier in minimally invasive surgery appears to be single-site robot-assisted surgery.

Single-site surgery is associated with a steep learning curve and technical difficulties such as the loss of port triangulation and the clashing of laparoscopic instruments. To overcome these hurtles and facilitate the implementation of laparoendoscopic single-site surgery, da Vinci single-site instruments and accessories have been introduced. A singlesite port, requiring a $2-3 \mathrm{~cm}$ incision, has multiple channels that provide access for two single-site Instruments, the $8.5 \mathrm{~mm}$ high-definition three-dimensional endoscope, a $5 \mathrm{~mm}$ or $10 \mathrm{~mm}$ accessory port, and an insufflation adaptor. Curved $5 \mathrm{~mm}$ instrument cannulas within the single-site port are designed to optimize triangulation toward the target anatomy while minimizing external collision of the instruments and camera arms. Semirigid instruments are placed into these curved cannulae, and they cross over each other within the port so that the instrument that enters on the right side becomes the left-sided operative instrument, and vice versa. This curved architecture sufficiently separates the instrument arms outside the body wall while maximizing the range of motion and minimizing instrument crowding intraperitoneally. ${ }^{2}$

Single-site robot-assisted surgery has been described in the literature and has been demonstrated to be safe and feasible in benign and malignant gynecologic surgeries. ${ }^{70-73}$
Although it may be feasible, a recent SGO survey found that only $5 \%$ of respondents indicated that single-site laparoscopy has an important or very important role in the field. ${ }^{1}$ Single-site surgery undoubtedly requires further training and certification. Whether single-site surgery proves to be relevant remains to be seen. The advantages afforded by robot-assisted surgery offer a greater chance of overcoming the cumbersome nature of single-site surgery compared to traditional laparoscopy.

\section{Conclusion}

The increase in the utilization of robot-assisted surgery in gynecological oncology is undeniable. Although the literature is lacking in large prospective studies, it has been demonstrated throughout this review article that patients appear to benefit from its utilization. Minimal invasive surgery undoubtedly has benefits over laparotomy, such as improved perioperative outcomes, shorter hospital stays, improved quality of life, and a faster return to daily functions and the workforce. ${ }^{6}$ Robot-assisted surgery has been a large catalyst in the increased use of minimally invasive surgery in managing gynecological cancers. One likely reason is that the learning curve for robot-assisted surgery is shorter than that for traditional laparoscopy. ${ }^{5}$ Additionally, robotassisted surgery offers technical advantages over traditional laparoscopy, such as high-definition three-dimensional field of vision, instruments with wrist-like range of motion, tremor filtration, and better ergonomics. ${ }^{7-9}$

Technology and surgical techniques will inevitably continue to improve hand in hand, thereby allowing procedures to be performed by methods that are even less invasive than today's standards. Furthermore, the cost of robot-assisted surgery will likely continue to decline as competition within the industry intensifies. Robot-assisted surgery will continue to have a place in the management of gynecological malignancies in the foreseeable future. While the FDA attempts to evaluate all new technology before granting approval for medical usage, we as physicians must nevertheless continue to assess the utility and feasibility of these technologies before implementing them into our clinical practice.

\section{Disclosure}

The authors report no conflicts of interest in this work.

\section{References}

1. Conrad LB, Ramirez PT, Burke W, et al. Role of minimally invasive surgery in gynecologic oncology: an updated survey of members of the society of gynecologic oncology. Int J Gynecol Cancer. 2015;25(6): 1121-1127. 
2. Surgical I. Investor $F A Q ; 2015$. Available from: http://phx.corporate-ir. net/phoenix.zhtml?c=122359\&p=irol-faq $\# 22324$. Accessed September 02, 2015.

3. Barbash GI, Glied SA. New technology and health care costs - the case of robot-assisted surgery. N Engl J Med. 2010;363(8):701-704.

4. Sfakianos GP, Frederick PJ, Kendrick JE, Straughn JM, Kilgore LC, Huh WK. Robotic surgery in gynecologic oncology fellowship programs in the USA: a survey of fellows and fellowship directors. Int $J$ Med Robot. 2010;6(4):405-412.

5. Ramirez PT, Adams S, Boggess JF, et al. Robotic-assisted surgery in gynecologic oncology: a Society of Gynecologic Oncology consensus statement. Developed by the Society of Gynecologic Oncology's Clinical Practice Robotics Task Force. Gynecol Oncol. 2012;124(2):180-184.

6. Sinno AK, Fader AN. Robotic-assisted surgery in gynecologic oncology. Fertil Steril. 2014;102(4):922-932.

7. Bell MC, Torgerson J, Seshadri-Kreaden U, Suttle AW, Hunt S. Comparison of outcomes and cost for endometrial cancer staging via traditional laparotomy, standard laparoscopy and robotic techniques. Gynecol Oncol. 2008;111(3):407-411.

8. Boggess JF, Gehrig PA, Cantrell L, et al. A comparative study of 3 surgical methods for hysterectomy with staging for endometrial cancer: robotic assistance, laparoscopy, laparotomy. Am J Obstet Gynecol. 2008;199(4):e361-e369.

9. ElSahwi KS, Hooper C, De Leon MC, et al. Comparison between 155 cases of robotic vs 150 cases of open surgical staging for endometrial cancer. Gynecol Oncol. 2012;124(2):260-264.

10. American Cancer Society. Cancer Facts and Figures 2015. Atlanta: American Cancer Society; 2015.

11. Pecorelli S. Revised FIGO staging for carcinoma of the vulva, cervix, and endometrium. Int J Gynaecol Obstet. 2009;105(2):103-104.

12. Walker JL, Piedmonte MR, Spirtos NM, et al. Laparoscopy compared with laparotomy for comprehensive surgical staging of uterine cancer: Gynecologic Oncology Group Study LAP2. J Clin Oncol. 2009;27(32) 5331-5336.

13. Walker JL, Piedmonte MR, Spirtos NM, et al. Recurrence and survival after random assignment to laparoscopy versus laparotomy for comprehensive surgical staging of uterine cancer: Gynecologic Oncology Group LAP2 Study. J Clin Oncol. 2012;30(7):695-700.

14. Chiou HY, Chiu LH, Chen CH, Yen YK, Chang CW, Liu WM. Comparing robotic surgery with laparoscopy and laparotomy for endometrial cancer management: a cohort study. Int J Surg. 2015;13:17-22.

15. Coronado PJ, Herraiz MA, Magrina JF, Fasero M, Vidart JA. Comparison of perioperative outcomes and cost of robotic-assisted laparoscopy, laparoscopy and laparotomy for endometrial cancer. Eur $J$ Obstet Gynecol Reprod Biol. 2012;165(2):289-294.

16. Jung YW, Lee DW, Kim SW, et al. Robot-assisted staging using three robotic arms for endometrial cancer: comparison to laparoscopy and laparotomy at a single institution. J Surg Oncol. 2010;101(2): $116-121$.

17. Corrado G, Cutillo G, Pomati G, et al. Surgical and oncological outcome of robotic surgery compared to laparoscopic and abdominal surgery in the management of endometrial cancer. Eur J Surg Oncol. 2015;41(8):1074-1081.

18. Vidal F, Rafii A. Lymph node assessment in endometrial cancer: towards personalized medicine. Obstet Gynecol Int. 2013;2013:892465.

19. Seamon LG, Bryant SA, Rheaume PS, et al. Comprehensive surgical staging for endometrial cancer in obese patients: comparing robotics and laparotomy. Obstet Gynecol. 2009;114(1):16-21.

20. Subramaniam A, Kim KH, Bryant SA, et al. A cohort study evaluating robotic versus laparotomy surgical outcomes of obese women with endometrial carcinoma. Gynecol Oncol. 2011;122(3):604-607.

21. Cardenas-Goicoechea J, Shepherd A, Momeni M, et al. Survival analysis of robotic versus traditional laparoscopic surgical staging for endometrial cancer. Am J Obstet Gynecol. 2014;210(2):e160-e161.

22. Seamon LG, Cohn DE, Henretta MS, et al. Minimally invasive comprehensive surgical staging for endometrial cancer: robotics or laparoscopy? Gynecol Oncol. 2009;113(1):36-41.
23. Seror J, Bats AS, Huchon C, Bensaid C, Douay-Hauser N, Lecuru F. Laparoscopy vs robotics in surgical management of endometrial cancer: comparison of intraoperative and postoperative complications. J Minim Invasive Gynecol. 2014;21(1):120-125.

24. Frey MK, Lin JF, Stewart LE, Makaroun L, Panico VJ, Holcomb K. Comparison of two minimally invasive approaches to endometrial cancer staging: a single-surgeon experience. J Reprod Med. 2015; 60(3-4):127-134.

25. Holtz DO, Miroshnichenko G, Finnegan MO, Chernick M, Dunton CJ. Endometrial cancer surgery costs: robot vs laparoscopy. J Minim Invasive Gynecol. 2010;17(4):500-503.

26. Wright JD, Herzog TJ, Neugut AI, et al. Comparative effectiveness of minimally invasive and abdominal radical hysterectomy for cervical cancer. Gynecol Oncol. 2012;127(1):11-17.

27. Sert MB, Abeler V. Robot-assisted laparoscopic radical hysterectomy: comparison with total laparoscopic hysterectomy and abdominal radical hysterectomy; one surgeon's experience at the Norwegian Radium Hospital. Gynecol Oncol. 2011;121(3):600-604.

28. Boggess JF, Gehrig PA, Cantrell L, et al. A case-control study of robot-assisted type III radical hysterectomy with pelvic lymph node dissection compared with open radical hysterectomy. Am J Obstet Gynecol. 2008;199(4):e351-e357.

29. Persson J, Reynisson P, Borgfeldt C, Kannisto P, Lindahl B, Bossmar T. Robot assisted laparoscopic radical hysterectomy and pelvic lymphadenectomy with short and long term morbidity data. Gynecol Oncol. 2009;113(2):185-190.

30. Asciutto KC, Kalapotharakos G, Lofgren M, Hogberg T, Borgfeldt C. Robot-assisted surgery in cervical cancer patients reduces the time to normal activities of daily living. Acta Obstet Gynecol Scand. 2015; 94(3):260-265.

31. Estape R, Lambrou N, Diaz R, Estape E, Dunkin N, Rivera A. A case matched analysis of robotic radical hysterectomy with lymphadenectomy compared with laparoscopy and laparotomy. Gynecol Oncol. 2009;113(3):357-361.

32. Ko EM, Muto MG, Berkowitz RS, Feltmate CM. Robotic versus open radical hysterectomy: a comparative study at a single institution. Gynecol Oncol. 2008;111(3):425-430.

33. Magrina JF, Kho RM, Weaver AL, Montero RP, Magtibay PM. Robotic radical hysterectomy: comparison with laparoscopy and laparotomy. Gynecol Oncol. 2008;109(1):86-91.

34. Tinelli R, Malzoni M, Cosentino F, et al. Robotics versus laparoscopic radical hysterectomy with lymphadenectomy in patients with early cervical cancer: a multicenter study. Ann Surg Oncol. 2011;18(9): 2622-2628.

35. Díaz-Feijoo B, Gil-Ibáñez B, Pérez-Benavente A, et al. Comparison of robotic-assisted vs conventional laparoscopy for extraperitoneal paraaortic lymphadenectomy. Gynecol Oncol. 2014;132(1): 98-101.

36. Soliman PT, Langley G, Munsell MF, Vaniya HA, Frumovitz M, Ramirez PT. Analgesic and antiemetic requirements after minimally invasive surgery for early cervical cancer: a comparison between laparoscopy and robotic surgery. Ann Surg Oncol. 2013;20(4):1355-1359.

37. Huang M, Slomovitz BM, Ramirez PT. Transperitoneal versus extraperitoneal para-aortic lymphadenectomy in patients with cervical cancer. Rev Obstet Gynecol. 2009;2(2):101-106.

38. Magrina JF, Kho R, Montero RP, Magtibay PM, Pawlina W. Robotic extraperitoneal aortic lymphadenectomy: development of a technique. Gynecol Oncol. 2009;113(1):32-35.

39. Vergote I, Pouseele B, Van Gorp T, et al. Robotic retroperitoneal lower para-aortic lymphadenectomy in cervical carcinoma: first report on the technique used in 5 patients. Acta Obstet Gynecol Scand. 2008;87(7): 783-787.

40. Hudry D, Ahmad S, Zanagnolo V, et al; SERGS Group. Robotically assisted para-aortic lymphadenectomy: surgical results: a cohort study of 487 patients. Int J Gynecol Cancer. 2015;25(3):504-511.

41. Lambaudie E, Narducci F, Leblanc E, et al. Robotically assisted laparoscopy for paraaortic lymphadenectomy: technical description and results of an initial experience. Surg Endosc. 2012;26(9):2430-2435. 
42. Magrina JF, Long JB, Kho RM, Giles DL, Montero RP, Magtibay PM. Robotic transperitoneal infrarenal aortic lymphadenectomy: technique and results. Int J Gynecol Cancer. 2010;20(1):184-187.

43. Noyes N, Abu-Rustum NR, Ramirez PT, Plante M. Options in the management of fertility-related issues after radical trachelectomy in patients with early cervical cancer. Gynecol Oncol. 2009;114(1):117-120.

44. Al-Niaimi AN, Einstein MH, Perry L, Hartenbach EM, Kushner DM. Uterine artery sparing robotic radical trachelectomy (AS-RRT) for early cancer of the cervix. Int J Gynaecol Obstet. 2011;112(1):76-80.

45. Burnett AF, Stone PJ, Duckworth LA, Roman JJ. Robotic radical trachelectomy for preservation of fertility in early cervical cancer: case series and description of technique. J Minim Invasive Gynecol. 2009;16(5):569-572.

46. Chuang LT, Lerner DL, Liu CS, Nezhat FR. Fertility-sparing robotic-assisted radical trachelectomy and bilateral pelvic lymphadenectomy in early-stage cervical cancer. J Minim Invasive Gynecol. 2008;15(6):767-770.

47. Geisler JP, Orr CJ, Manahan KJ. Robotically assisted total laparoscopic radical trachelectomy for fertility sparing in stage IB1 adenosarcoma of the cervix. J Laparoendosc Adv Surg Tech A. 2008;18(5):727-729.

48. Mejia-Gomez J, Feigenberg T, Arbel-Alon S, Kogan L, Benshushan A. Radical trachelectomy: a fertility-sparing option for early invasive cervical cancer. Isr Med Assoc J. 2012;14(5):324-328.

49. Nick AM, Frumovitz MM, Soliman PT, Schmeler KM, Ramirez PT. Fertility sparing surgery for treatment of early-stage cervical cancer: open vs robotic radical trachelectomy. Gynecol Oncol. 2012;124(2): 276-280.

50. Persson J, Imboden S, Reynisson P, Andersson B, Borgfeldt C, Bossmar T. Reproducibility and accuracy of robot-assisted laparoscopic fertility sparing radical trachelectomy. Gynecol Oncol. 2012;127(3): 484-488.

51. Ramirez PT, Schmeler KM, Malpica A, Soliman PT. Safety and feasibility of robotic radical trachelectomy in patients with early-stage cervical cancer. Gynecol Oncol. 2010;116(3):512-515.

52. Vitobello D, Siesto G, Bulletti C, Accardi A, Ieda N. Robotic radical parametrectomy with pelvic lymphadenectomy: our experience and review of the literature. Eur J Surg Oncol. 2012;38(6):548-554.

53. Ramirez PT, Schmeler KM, Wolf JK, Brown J, Soliman PT. Robotic radical parametrectomy and pelvic lymphadenectomy in patients with invasive cervical cancer. Gynecol Oncol. 2008;111(1):18-21.

54. Magrina JF, Magtibay PM. Robotic nerve-sparing radical parametrectomy: feasibility and technique. Int J Med Robot. 2012;8(2):206-209.

55. Katz VL. Comprehensive Gynecology. 5th ed. Philadelphia: Mosby Elsevier; 2007.

56. Lim PC. Robotic assisted total pelvic exenteration: a case report. Gynecol Oncol. 2009;115(2):310-311.

57. Davis MA, Adams S, Eun D, Lee D, Randall TC. Robotic-assisted laparoscopic exenteration in recurrent cervical cancer Robotics improved the surgical experience for 2 women with recurrent cervical cancer. Am J Obstet Gynecol. 2010;202(6):663e661.
58. Puntambekar S, Lawande A, Desai R, Puntambekar S, Joshi GA, Joshi SN. Initial experience of robotic anterior pelvic exenteration at a single institute. Int J Gynaecol Obstet. 2014;126(1):41-44.

59. Iavazzo C, Gkegkes ID. Robotic technology for pelvic exenteration in cases of cervical cancer. Int J Gynaecol Obstet. 2014;125(1):15-17.

60. Wright JD, Ananth CV, Tergas AI, et al. An economic analysis of robotically assisted hysterectomy. Obstet Gynecol. 2014;123(5):1038-1048.

61. Reynisson P, Persson J. Hospital costs for robot-assisted laparoscopic radical hysterectomy and pelvic lymphadenectomy. Gynecol Oncol. 2013;130(1):95-99.

62. Chen $\mathrm{CH}$, Chiu LH, Chen HH, Chan C, Liu WM. Comparison of robotic approach, laparoscopic approach and laparotomy in treating epithelial ovarian cancer. Int J Med Robot. Epub 2015 Mar 25.

63. Feuer GA, Lakhi N, Barker J, Salmieri S, Burrell M. Perioperative and clinical outcomes in the management of epithelial ovarian cancer using a robotic or abdominal approach. Gynecol Oncol. 2013;131(3): $520-524$.

64. Magrina JF, Zanagnolo V, Noble BN, Kho RM, Magtibay P. Robotic approach for ovarian cancer: perioperative and survival results and comparison with laparoscopy and laparotomy. Gynecol Oncol. 2011; 121(1):100-105.

65. Sinno AK, Fader AN, Roche KL, Giuntoli RL 2nd, Tanner EJ. A comparison of colorimetric versus fluorometric sentinel lymph node mapping during robotic surgery for endometrial cancer. Gynecol Oncol. 2014;134(2):281-286.

66. Jewell EL, Huang JJ, Abu-Rustum NR, et al. Detection of sentinel lymph nodes in minimally invasive surgery using indocyanine green and nearinfrared fluorescence imaging for uterine and cervical malignancies. Gynecol Oncol. 2014;133(2):274-277.

67. Rossi EC, Ivanova A, Boggess JF. Robotically assisted fluorescenceguided lymph node mapping with ICG for gynecologic malignancies: a feasibility study. Gynecol Oncol. 2012;124(1):78-82.

68. Levinson KL, Auer M, Escobar PF. Evolving technologies in robotic surgery for minimally invasive treatment of gynecologic cancers. Expert Rev Med Devices. 2013;10(5):603-610.

69. Holloway RW, Bravo RA, Rakowski JA, et al. Detection of sentinel lymph nodes in patients with endometrial cancer undergoing roboticassisted staging: a comparison of colorimetric and fluorescence imaging. Gynecol Oncol. 2012;126(1):25-29.

70. Akdemir A, Yildirim N, Zeybek B, Karaman S, Sendag F. Single incision trans-umbilical total hysterectomy: robotic or laparoscopic? Gynecol Obstet Invest. Epub 2015 Jan 27.

71. Cela V, Freschi L, Simi G, Ruggiero M, Tana R, Pluchino N. Robotic single-site hysterectomy: feasibility, learning curve and surgical outcome. Surg Endosc. 2013;27(7):2638-2643.

72. Sinno AK, Fader AN, Tanner EJ 3rd. Single site robotic sentinel lymph node biopsy and hysterectomy in endometrial cancer. Gynecol Oncol. 2015;137(1):190

73. Yoo HN, Kim TJ, Lee YY, et al. Single-site robotic surgery in gynecologic cancer: a pilot study. J Gynecol Oncol. 2015;26(1):62-67.
Robotic Surgery: Research and Reviews

\section{Publish your work in this journal}

Robotic Surgery: Research and Reviews is an international, peer reviewed, open access, online journal publishing original research, commentaries, reports, and reviews on the theory, use and application of robotics in surgical interventions. Articles on the use of supervisory-controlled robotic systems, telesurgical devices, and shared-control systems are

\section{Dovepress}

invited. The manuscript management system is completely online and includes a very quick and fair peer review system, which is all easy to use. Visit http://www.dovepress.com/testimonials.php to read real quotes from published authors. 